\title{
Analysis of Clinicopathologic Factors and KRAS Gene Mutation in Epithelial Ovarian Cancer Outcomes
}

\author{
Andi Friadi ${ }^{1 *}$, Wirsma Arif Harahap ${ }^{2}$, Arni Amir $^{3}$, Andri Andrijono ${ }^{4}$ \\ ${ }^{1}$ Department of Obstetrics and Gynecology, Faculty of Medicine, Andalas University, Padang, Indonesia; ${ }^{2}$ Department of \\ Surgery, Faculty of Medicine, Andalas University, Padang, Indonesia; ${ }^{3}$ Department of Biology, Faculty of Medicine, Andalas \\ University, Padang, Indonesia; ${ }^{4}$ Department of Obstetrics and Gynecology, Faculty of Medicine, University of Indonesia, \\ Jakarta, Indonesia
}

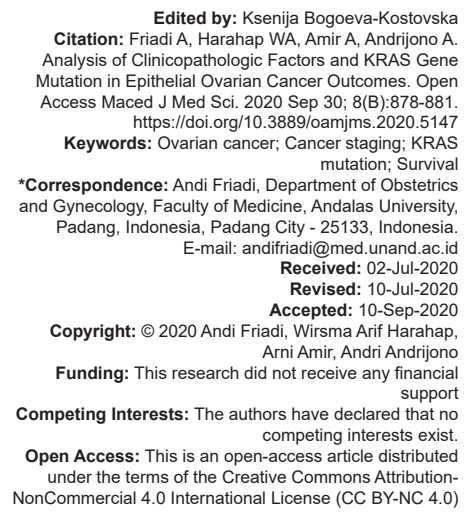

\section{Introduction}

Ovarian cancer has a high incidence and mortality rate in the world. Deaths due to ovarian cancer are caused by the recurrence of ovarian cancer. This is inseparable from various prognostic factors associated with recurrence, such as staging, grading and which is currently the center of attention by many experts that are gene mutation. One of the gene mutations that have been studied in cancer pathophysiology is the Kirstenrat sarcoma virus oncogene (KRAS) [1], [2]. Under normal conditions, KRAS is a proto-oncogene that is involved in the signaling pathway that regulates various important cellular functions such as proliferation, growth, and motility [3], [4].

There is controversy among some experts who analyze the ovarian cancer prognosis associated with KRAS mutation. One researcher declared that KRAS mutation can occur at an early stage of ovarian cancer and had a good prognosis, on the other hand, there were researchers who reported that the KRAS mutation was associated with high recurrence rate and poor prognosis [5], [6]. The aim of this study is to prove that staging, grading, and KRAS exon 2 mutations will influence recurrence in epithelial ovarian cancer.

\section{Materials and Methods}

\section{Study design and research samples}

The authors conducted a retrospective cohort study. Clinicopathological factors and prognoses were obtained for 205 patients who were histopathologically diagnosed with epithelial ovarian cancer or ovarian borderline malignant tumor, operated from June 2015 to January 2019 at Dr. M. Djamil General Hospital. Approval was obtained from the Ethics Boards of Andalas University 
(approval No. 2072/KEP/FK/2020) and Dr. M. Djamil General Hospital (approval No. 86/KEPK/2020). Among the 205 patients, those with missing prognostic data were excluded, leaving 80 patients for analysis, including 40 patients with mucinous ovarian cancer and 40 patients with non-mucinous ovarian cancer.

\section{Data collection technique}

Staging were classified based on FIGO 2014 which were divided into early stage (stage IC2-IIA) and advanced stage (Stage IIB-IVB). Grading was classified based on Shimizu-Silverberg criteria. All slides were reviewed for grading by one pathologist. KRAS mutation was classified into mutation and non-mutation based on sequencing. Regarding the recurrence, patients were followed up to see whether there was a recurrence based on the presence or absence of complaints and ultrasound examination. Because of COVID-19 pandemic, patients were followed up only by phone call.

Regarding the histological type, serous carcinoma + endometrial carcinoma + clear cell carcinoma was defined as the non-mucinous group, and mucinous carcinoma as the mucinous group for analysis because it is known that non-mucinous group cancer is often less frequent KRAS gene mutation, whereas mucinous group cancer is often more frequent KRAS gene mutation [5].

\section{Data analysis}

Clinicopathological factors and KRAS mutation were analyzed by $\chi^{2}$ test multivariate analysis by logistic regression. Survival rate was determined using the Kaplan-Meier method and examined by Log rank test. All analyses were performed using STATA ver. 12.0 (StataCorp, 4905 Lakeway Drive College Station, Texas, USA), with $p<0.05$ considered to be significant.

\section{Results}

Characteristic of the study subjects (Table 1).

Table 1: Characteristics of the study subjects

\begin{tabular}{lll}
\hline Characteristics & $f / \%$ & Median (Min-Max) \\
\hline Age (years) & & $48(24-80)$ \\
Observation period (months) & & 19.88 \\
Stage & $35(43.75)$ & \\
Stage I & $12(15.00)$ & \\
Stage II & $27(33.75)$ & \\
Stage IIIB & $6(7.50)$ & \\
Stage IV & & \\
Grading & $33(41.25)$ & \\
Low grade & $47(58.75)$ & \\
High grade & & \\
Histological classification & $40(50.00)$ & \\
$\quad$ Mucinous carcinoma & $25(31.25)$ & \\
Serous carcinoma & $10(12.50)$ & \\
$\quad$ Clear cell carcinoma & $5(6.25)$ & \\
Endometrioid carcinoma & & \\
\hline
\end{tabular}

Table 1 identified that the median age of the patients was 48 years (range: $24-80$ years). The median observation period was 19.88 months. In the FIGO 2014 classification, 35 patients were classified as Stage I (29 as Stage IC2 and 6 as Stage IC3), 12 as Stage II (4 as Stage IIA and 8 as Stage IIB), and 27 as Stage IIIB, and 6 as Stage IV (4 as Stage IVA and 2 as Stage IVB). In the histological classification, 40 cases were classified as mucinous carcinoma, 25 as serous carcinoma, ten as clear cell carcinoma, and five as endometrioid carcinoma. In two-tier grading system (Shimizu-Silverberg), 33 cases were classified as low grade and 47 as high grade.

Comparison of 2-year disease-free survival rates between non-mutation KRAS and mutation KRAS gene (Table 2).

Table 2: Comparison of 2-year disease-free survival rates between non-mutation KRAS and mutation KRAS gene

\begin{tabular}{|c|c|c|c|c|c|c|c|}
\hline \multirow[t]{2}{*}{ KRAS exon 2} & \multirow[t]{2}{*}{$\mathrm{n}$} & \multirow{2}{*}{$\begin{array}{l}\begin{array}{l}\text { Median } \\
\text { (months) }\end{array} \\
\end{array}$} & \multicolumn{2}{|c|}{ Survival rate (\%) } & \multicolumn{3}{|c|}{ Cox-regression } \\
\hline & & & 12 months & 24 months & $\mathrm{HR}$ & $95 \% \mathrm{Cl}$ & $p$ \\
\hline Non-mutation & 61 & 22.5 & 70.49 & 47.58 & Ref & - & - \\
\hline Mutation & 19 & 13.9 & 57.89 & 31.56 & 2.086 & $1.14-3.79$ & $0.01^{*}$ \\
\hline
\end{tabular}

Table 2 shows that the 2-year disease-free survival rates in KRAS group were $47.58 \%$ in nonmutation and $31.56 \%$ in mutation, with significant differences between two groups (Figure 1).

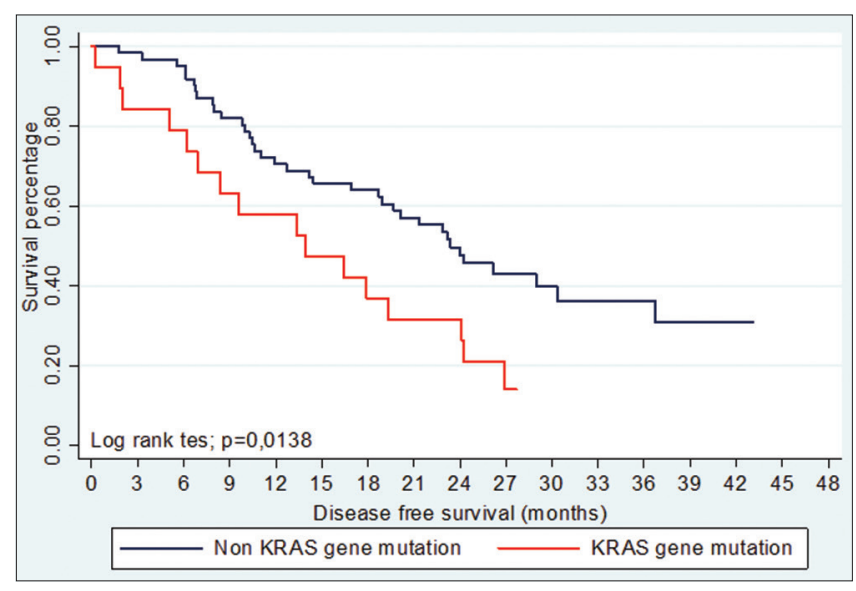

Figure 1: Kaplan-Meier comparison of 2-year disease-free survival rates between non-mutation KRAS and mutation KRAS gene

Comparison of 2-year disease-free survival rates between staging and mutation KRAS gene (Table 3).

Table 3: Comparison of 2-year disease-free survival rates between staging and mutation KRAS gene

\begin{tabular}{|c|c|c|c|c|c|c|c|}
\hline \multirow{2}{*}{$\begin{array}{l}\text { Staging and } \\
\text { KRAS gene }\end{array}$} & \multirow[t]{2}{*}{$\mathrm{n}$} & \multirow{2}{*}{$\begin{array}{l}\text { Median } \\
\text { (months) }\end{array}$} & \multicolumn{2}{|c|}{ Survival rate (\%) } & \multicolumn{3}{|c|}{ Cox-regression } \\
\hline & & & 12 months & 24 months & $\overline{\mathrm{HR}}$ & $95 \% \mathrm{Cl}$ & $\mathrm{p}$ \\
\hline $\begin{array}{l}\text { Early stage-non } \\
\text { mutation }\end{array}$ & 30 & 25,5 & 93,33 & 85,79 & Ref & - & - \\
\hline $\begin{array}{l}\text { Early stage- } \\
\text { KRAS mutation }\end{array}$ & 9 & 17,9 & 88,89 & 44,44 & 3,632 & $1,26-10,42$ & 0,02 \\
\hline $\begin{array}{l}\text { Advanced stage- } \\
\text { non mutation }\end{array}$ & 31 & 11,9 & 48,39 & 10,65 & 7,116 & $3,25-15,53$ & 0,00 \\
\hline $\begin{array}{l}\text { Advanced stage - } \\
\text { KRAS mutation }\end{array}$ & 10 & 6,5 & 30,00 & 20,00 & 11,413 & $4,51-28,87$ & 0,00 \\
\hline
\end{tabular}

Table 3 known that the 2-year disease-free survival rates (2y-DFS) in non-mutation group were $85.79 \%$ in early stage, and $10.65 \%$ in advanced stage. 
The 2-year $2 y$-DFS in mutation group were $44.44 \%$ in early stage, and $20.00 \%$ in advanced stage with significant differences between early stage with nonmutation and advanced stage with mutation $(p=0.00)$ (Figure 2).

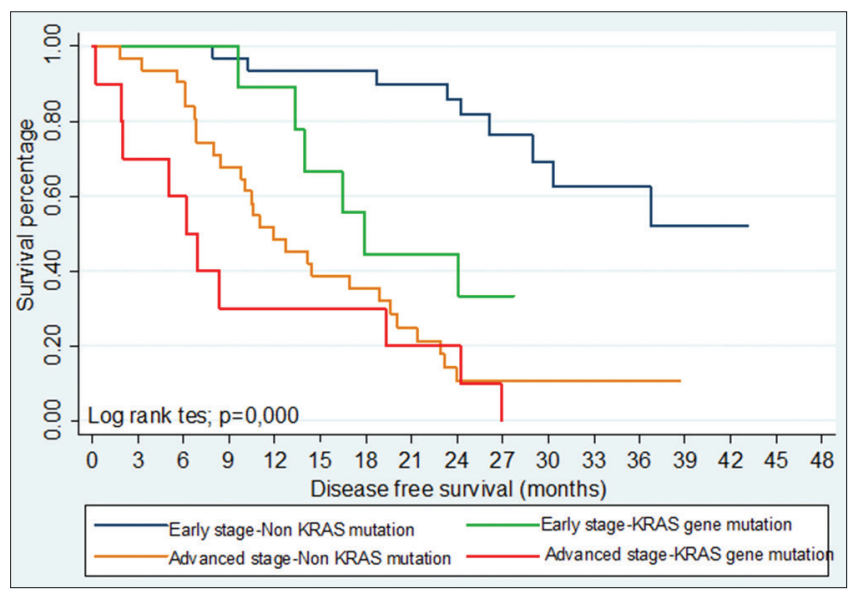

Figure 2: Kaplan-Meier comparison of 2-year disease-free survival rates between staging and mutation KRAS gene

\section{Discussion}

In this study, we analyzed that clinicopathologicmolecular prognostic factors (staging-grading-KRAS exon 2 mutation) are factors that cause an increase in ovarian cancer recurrence. Recurrence of ovarian cancer associated significantly with staging, grading, and KRAS exon 2 mutations.

There is a significant difference in the diseasefree survival of KRAS exon 2 mutations compared to non-mutation. Within 2 years, there was a decrease in survival by $68.44 \%$ in group with KRAS exon 2 mutations. There was a significant difference in diseasefree survival in the advanced stage ovarian cancer group with the KRAS exon 2 mutations. There was a decrease in survival by $80 \%$ in the group advanced stage with KRAS exon 2 mutations in an interval of 2 years.

Our data supported that the KRAS exon 2 mutations are very important on disease-free survival. Previous study reported that the survival of ovarian cancer with KRAS mutations is shorter compared to without mutations $(p<0.05)$ [7]. Another study known, that cancer survival pulmonary mutations with KRAS mutations are shorter than those without mutations [8].

The clinical aspect in this study is the importance of the recommendation to ask patients to come up every 3 months regularly for 2 years postoperatively, especially in patients who have the KRAS exon 2 mutations. Previously, it is recommended to follow-up every 6 months after the first year, so with the results of this study are expected to follow-up patients remain 3 months for the $2^{\text {nd }}$ year, not 6 months. This is useful to detect early if there is a recurrence in patients with advanced ovarian cancer with the KRAS exon 2 mutations.

The results of this study concluded that KRAS exon 2 mutations and prognostic factors significantly influenced recurrence in epithelial ovarian cancer. Since this is retrospective study that used 80 ovarian cancer tissues operated from June 2015 to January 2019, grading was reviewed by one pathologist using two-tier system especially for mucinous carcinoma, sequencing by Sanger technique to assess exon 2 mutations. Based on this sequencing, we found patients with multiple mutations.

In addition, recurrences were recorded by medical history of a patient and ultrasound examination. Therefore, DFS was analyzed for prognosis in this study. Despite these findings, this study has limitation that patients were followed up for recurrence only by phone call due to COVID-19 pandemic.

There was a significant difference in 2-year DFS among non-mutation and KRAS mutation group, suggesting that both groups clearly reflect the prognosis of ovarian cancer. Regarding combination staging and KRAS mutation, there were significant differences in 2y-DFS between early stage without mutation and early stage with mutation, advanced stage without mutation and advanced stage with mutation. In our study, prognosis in early stage without KRAS mutation differed significantly compared to early stage with mutation, but those in advanced stages without and with mutation were similar. The prognosis did not differ significantly between without mutation and with KRAS mutation in the advanced stage group, but was significantly different in the early stage group, and thus we believe that KRAS mutation is important prognostic factors, especially in early stage ovarian cancer [9].

The previous study found survival in the early stages (Stage I-II), there were differences in survival between groups with KRAS mutations compared to groups without mutations, but in the advanced stage group (Stage III-IV) there were no differences in survival between groups with KRAS mutations compared to groups without mutations $(p>0.05)$ [7]. Another research conducted the effect of staging and classification of type 1 and type 2 ovarian cancers on disease-free survival. Researcher reported that the highest survival was the type 1 ovarian cancer group with an early stage and the lowest survival was the type II ovarian group with advanced stage. This shows the role of staging greatly influences recurrence free survival [10].

In this study suggested the important in clinical aspects, especially in asking patients with advanced stage and KRAS mutation exon 2 for regular follow-up every 2 months in 1 year postoperatively. To detect a recurrence early so that it can be managed faster. 


\section{Conclusion}

The results of this study concluded that KRAS exon 2 mutations and clinicopathologic factors significantly influenced the recurrence in epithelial ovarian cancer.

\section{Acknowledgments}

The authors would like to thank all patients and staff of Doctoral Program of Biomedicine, Faculty of Medicine, Andalas University, Padang City, Indonesia.

\section{References}

1. Rankin EB. Genomics and molecular mechanisms of high grade serous ovarian cancer: The $12^{\text {th }}$ biennial rivkin center ovarian cancer research symposium. Int J Gynecol Cancer. 2019;29(2):S7-11. https://doi.org/10.1136/ijgc-2019-000490

PMid:31462542

2. Erol A, Niemira M, Kretowski AJ. Novel approaches in ovarian cancer research against heterogeneity, late diagnosis, drug resistance, and transcoelomic metastases. Int $\mathrm{J} \mathrm{Mol} \mathrm{Sci.}$ 2019;20(11):2649. https://doi.org/10.3390/ijms20112649 PMid:31146417

3. Carta C, Pantaleoni F, Bocchinfuso G, Stella L, Vasta I, Sarkozy A, et al. Germline missense mutations affecting KRAS Isoform B are associated with a severe noonan syndrome phenotype. Am J Hum Genet. 2006;79(1):129-35. https://doi. org/10.1086/504394

\section{PMid: 16773572}

4. Murugan AK, Grieco M, Tsuchida N. RAS mutations in human cancers: Roles in precision medicine. Semin Cancer Biol. 2019;59:23-35. https://doi.org/10.1016/j. semcancer.2019.06.007

PMid:31255772

5. Hobbs GA, Der CJ. RAS mutations are not created equal. Cancer Discov. 2019;9(6):696-8. https://doi.org/10.1158/21598290.cd-19-0406

PMid:31160330

6. Khurana E, Fu Y, Chakravarty D, Demichelis F, Rubin MA Gerstein M. Role of non-coding sequence variants in cancer Nat Rev Genet. 2016;17:93-108. https://doi.org/10.1038/ nrg.2015.17

PMid:26781813

7. Nodin B, Zendehrokh N, Sundstrom M, Jirstrom $K$ Clinicopathological correlates and prognostic significance of KRAS mutation status in a pooled prospective cohort of epithelial ovarian cancer. Diagn Pathol. 2013;8:106. https://doi. org/10.1186/1746-1596-8-106

\section{PMid:23800114}

8. Zhou H, Dai Y, Zhu L, Wang C, Fei X, Pan Q, et al. Poo response to platinum-based chemotherapy is associated with KRAS mutation and concomitant low expression of BRAC1 and TYMS in NSCLC. J Int Med Res. 2016;44:89-98. https://doi. org $/ 10.1177 / 0300060515607383$

\section{PMid:26740498}

9. Pavlik EJ, Smith C, Dennis TS, Harvey E, Huang B, Chen Q, et al. Disease-specific survival of Type I and Type II epithelial ovarian cancers-stage challenges categorical assignments of indolence and aggressiveness. Diagnostics (Basel). 2020;10(2):56. https://doi.org/10.3390/diagnostics10020056 PMid:31973035

10. Prat J. Ovarian, fallopian tube and peritoneal cancer staging Rationale and explanation of new FIGO staging 2013. Best Pract Res Clin Obstet Gynaecol. 2015;29(6):858-69. https://doi. org/10.1016/j.bpobgyn.2015.03.006

PMid:25890882 